\title{
Classification of ECG ARRHYTHMias USING DISCRETE WAVELET TRANSFORM AND NEURAL NETWORKS
}

\author{
Maedeh Kiani Sarkaleh and Asadollah Shahbahrami \\ Department of Computer Engineering, Faculty of Engineering \\ P.O.Box: 3756-41635 \\ University of Guilan, Rasht, Iran \\ shahbahrami@guilan.ac.ir
}

\begin{abstract}
Automatic recognition of cardiac arrhythmias is important for diagnosis of cardiac abnormalies. Several algorithms have been proposed to classify ECG arrhythmias; however, they cannot perform very well. Therefore, in this paper, an expert system for ElectroCardioGram (ECG) arrhythmia classification is proposed. Discrete wavelet transform is used for processing ECG recordings, and extracting some features, and the Multi-Layer Perceptron (MLP) neural network performs the classification task. Two types of arrhythmias can be detected by the proposed system. Some recordings of the MIT-BIH arrhythmias database have been used for training and testing our neural network based classifier. The simulation results show that the classification accuracy of our algorithm is $96.5 \%$ using 10 files including normal and two arrhythmias.
\end{abstract}

\section{KEYWORDS}

ECG, Arrhythmia, Daubechies Wavelets, Discrete Wavelet Transform (DWT), Neural Network (NN).

\section{INTRODUCTION}

The ElectroCardioGram (ECG) signal is an important signal among all bioelectrical signals. Analysis of the ECG signal is widely used in the diagnosis of many cardiac disorders. It can be recorded from the wave passage of the depolarization and repolarization processes in the heart. The potential in the heart tissues is conducted to the body surface where it is measured using electrodes.

Figure 1 illustrates two periods of the normal ECG signal. The P, Q, R, S and T waves are the most important characteristic features of the ECG. The peaked area in the ECG beat, commonly called QRS complex, together with its neighboring $\mathrm{P}$ wave and $\mathrm{T}$ wave, is the portion of the signal through to contain most of the diagnostically important information. Other important information includes the elevation of the ST segment and heartbeat rate, the RR or PP. 


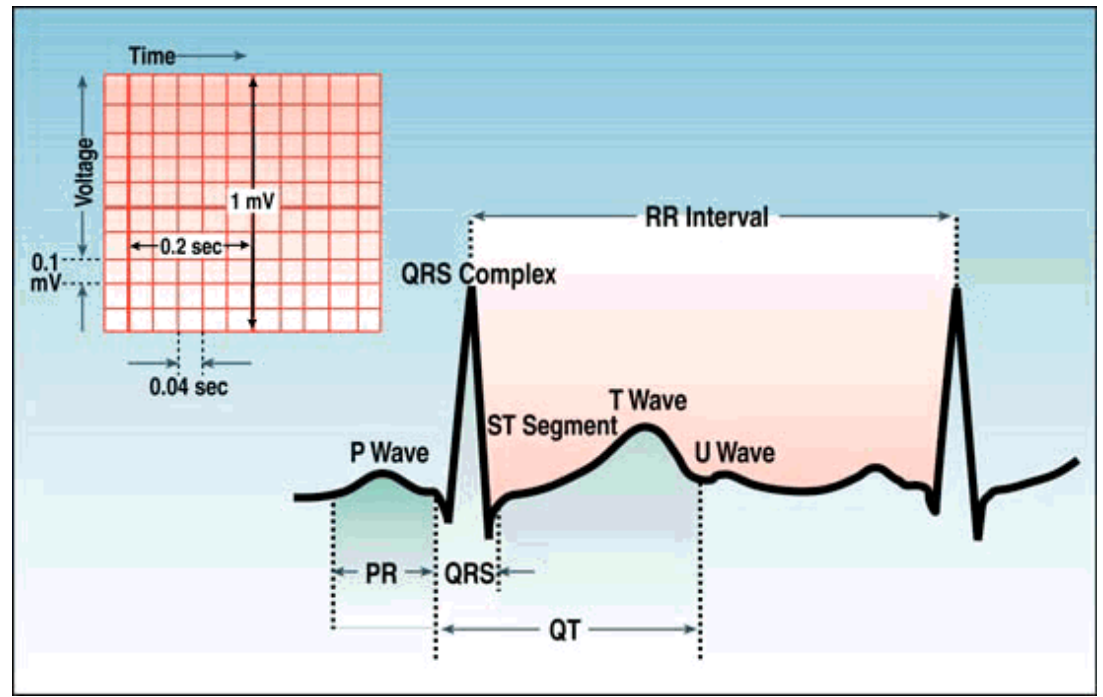

Figure 1. Two periods of the normal ECG signal [33].

The shape of ECG conveys very important hidden information in its structure. The amplitude and duration of each wave in ECG signals are often used for the manual analysis. Thus, the volume of the data being enormous and the manual analysis is tedious and very time-consuming task. Naturally, the possibility of the analyst missing vital information is high. Therefore, medical diagnostics can be performed using computer-based analysis and classification techniques [1].

Several algorithms have been proposed to classify ECG heartbeat patterns based on the features extracted from the ECG signals. Fourier transform analysis provides the signal spectrum or range of frequency amplitudes within the signal; however, Fourier transform only provides the spectral components, not their temporal relationships. Wavelets can provide a time versus frequency representation of the signal and work well on non-stationary data [2-4]. Other algorithms use morphological features [5], heartbeat temporal intervals [6], frequency domain features and multifractal analysis [7]. Biomedical signal processing algorithms require appropriate classifiers in order to best categorize different ECG signals. In 1976, Shortliffe presented an early computeraided diagnostic system for diagnosis and treatment of symptoms of bacterial infection [8]. Classification techniques for ECG patterns include linear discriminate analysis [2], support vector machines [9], artificial neural networks [10-14], mixture-of-experts algorithms [12], and statistical Markov models $[15,16]$. In addition, unsupervised clustering of the ECG signal has been performed using self-organizing maps in [17].

Besides the fact the ECG record can be noisy, the main problem in computer-based classification is the wide variety in the shape of beats belonging to the same class and beats of similar shape belonging to different classes $[18,19]$. Computer-based diagnosis algorithms have generally three steps, namely: EGC beat detection, extraction of useful features from beats, and classification.

In this paper, we propose a Neural Network (NN) based algorithm for classification of Paced Beat (PB), Artial Premature Beat (APB) arrhythmias as well as the normal signal. Our algorithm uses some features obtained by the Discrete Wavelet Transform (DWT) along with timing interval features to train an MLP NN. We extract some important features from the wavelet coefficients to achieve both an accurate and a robust NN based classifier by using a number of training patterns. 
This paper is organized as follows. Section 2 describes the background information about DWT and Artificial Neural Networks (ANNs). The proposed feature extraction technique as well as our classification system is present in Section 3. Section 4 explains the experimental results. Conclusions are drawn in Section 5.

\section{BACKGROUND}

\subsection{Discrete Wavelet Transform}

The wavelet transform was presented at the beginning of the 1980s by Morlet, who used it to evaluate seismic data [16]. Wavelets provide an alternative to classical Fourier algorithms for one and multi-dimensional data analysis and synthesis, and have numerous applications such as in mathematics, physics, and digital image processing. The wavelet transform can be applied in both continuous-time signal and discrete-time signal. For example, The wavelet representation of a discrete signal $\mathrm{X}$ consisting of $\mathrm{N}$ samples can be computed by convolving $\mathrm{X}$ with the Low-Pass Filters (LPF) and High-Pass Filters (HPF) and down-sampling the output signal by 2, so that the two frequency bands each contains N/2 samples.

This technique is based on the use of wavelets as the basis functions for representing other functions. These basis functions have a finite support in time and frequency domain. Multiresolution analysis is achieved by using the mother wavelet, and a family of wavelets generated by translations and dilations of it [20,21]. There are different approaches to implement the 2D DWT such as traditional convolution-based and lifting scheme methods. The convolutional algorithms apply filtering by multiplying the filter coefficients with the input samples and accumulating the results. These algorithms are implemented using Finite Impulse Response (FIR) filter banks. The lifting scheme has been proposed for the efficient implementation of the wavelet transform. This approach has three phases, namely: split, predict, and update [22, 23, 24]. In onedimensional DWT, at each decomposition level, the HPF associated with scaling function produces detail information which is related to high-frequency components, while the LPF associated with scaling function produces coarse approximations, which are related to lowfrequency components of the signal. The approximation part can be iteratively decomposed. This process for two-level decomposition is depicted in Figure 2. A signal is broken down into many lower resolution components. This operation is called the wavelet decomposition tree [24].

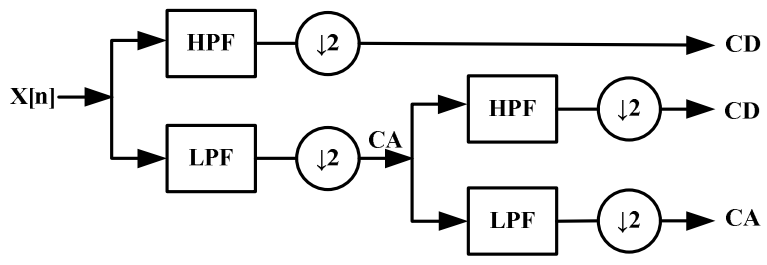

Figure 2. Sub-band decomposition of discrete wavelet transform implementation.

The wavelet transform is reversible. The reconstruction is the reverse process of decomposition. The approximation and detail wavelet coefficients at every level are up sampled by two, passed through the LPF and HPF and then added. This process is continued through the same number of levels as in the decomposition process to obtain the original signal. Figure 3 depicts this process. 


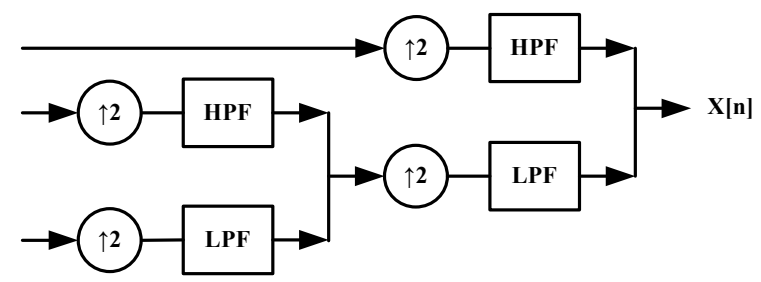

Figure 3. Wavelet reconstruction process.

Various wavelet families are defined in the literature. Daubechies wavelets are the most popular wavelets. The Daubechies wavelets are used in different applications. The wavelets filters are selected based on their ability to analyze the signal and their shape in an application. Figure 4 shows nine members of the Daubechies family.

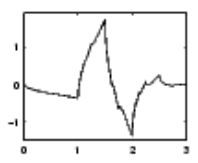

$\mathrm{db} 2$

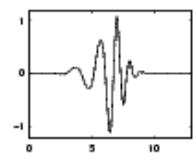

$\mathrm{db} 7$

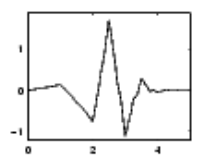

$\mathrm{db} 3$

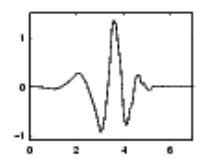

$\mathrm{db} 4$

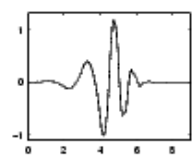

db5

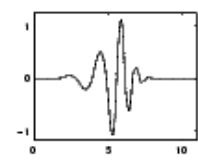

db6

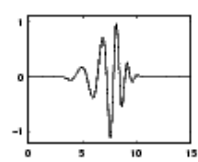

$\mathrm{db} 8$

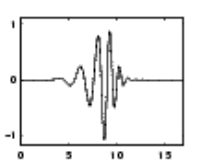

db9

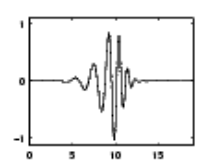

$\mathrm{db} 10$

Figure 4. Nine members of the Daubechies family.

The ECG signals are considered as representative signals of cardiac physiology, useful in diagnosing cardiac disorders. The most complete way to display this information is to perform spectral analysis. The wavelet transform provides very general technique which can be applied to many signal processing applications. Different features can be computed and manipulated in compressed domain using wavelet coefficients. All these means that we can apply the wavelet transform on the ECG signal and convert it to the wavelet coefficients or parameters. The obtained coefficients characterize the behavior of the ECG signal and the number of these coefficients are small than the number of the original signal. This reduction of feature space is particularly important for recognition and diagnostic purposes [25].

\subsection{Artificial Neural Networks}

The Artificial Neural Networks (ANN) are the tools, which can be used to model human cognition or neural biology using mathematical operations. An ANN is a processing element. It has has certain performance characteristics in common with biological neural networks. A neural network is characterized by 1) its pattern of connections between the neurons (called its architecture), 2) its algorithm of determining the weights on the connections (called its training, or learning algorithm), and 3) its activation function [26]. The MultiLayer Perceptron (MLP) is the most common neural network. This type of neural network is known as a supervised network because it requires a desired output in order to learn. The purpose of the MLP is to develop a model that correctly maps the input data to the output using historical data so that the model can 
then be used to produce the output result when the desired output is unknown. A graphical representation of an MLP is shown in Figure 5.

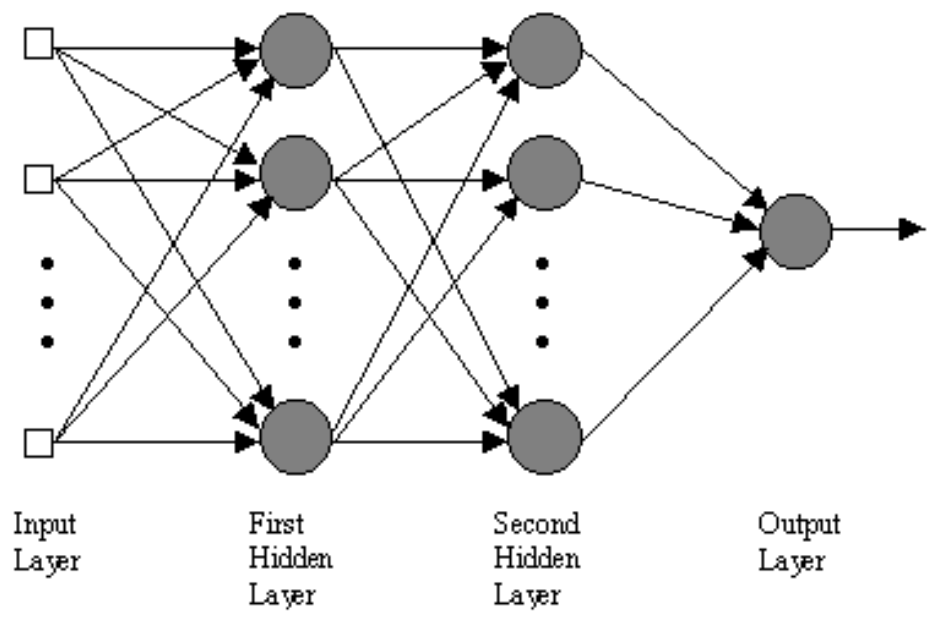

Figure 5. MLP architecture with two hidden layers [34].

In the first step, the MLP is used to learn the behaviour of the input data using back-propagation algorithm. This step is called the training phase. In the second step, the trained MLP is used to test using unknown input data. The back-propagation algorithm compares the result that is obtained in this step with the result that was expected. This kind of classification is called supervised classification. The MLP computes the error signal using the obtained output and desired output. The computed signal error is then fed back to the neural network and used to adjust the weights such that with each iteration the error decreases and the neural model gets closer and closer to produce the desired output. Figure 6 shows this process [24].

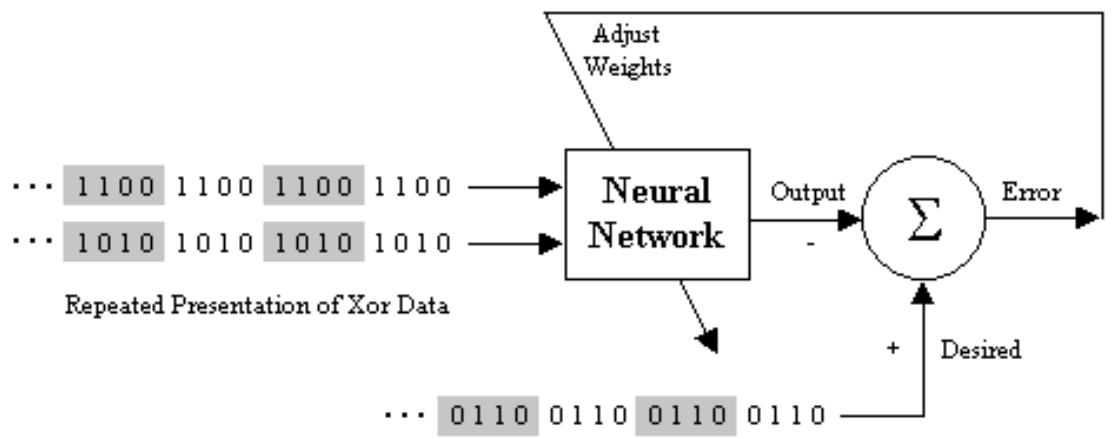

Figure 6. Neural network learning algorithm [34].

There are different training algorithms, while it is very difficult to know which training algorithm is the fastest for a given problem. In order to determine the fastest training algorithm, many parameters should be considered. For instance, the complexity of the problem, the number of data points in the training set, the number of weights, and biases in the network, the and error goal should be evaluated. 


\section{Proposed Classification System}

Figure 7 shows the block diagram of the proposed system. The system is based on wavelet transform and neural networks. This system consists of two phases: the feature extraction phase and the classification phase which are discussed in the following sections.

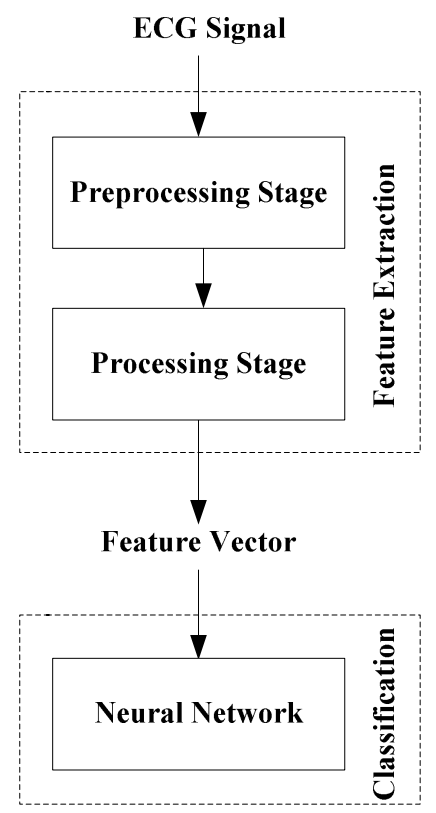

Figure 7. Block diagram of the proposed classification system.

\subsection{Feature Extraction Phase}

The first phase consists of two stages: preprocessing stage and processing stage. The preprocessing stage improves the classification accuracy of any algorithm; because, it gives us more accurate features.

The obtained ECG from body electrodes has the baseline noise. Baseline wander, which may appear due to a number of factors arising from biological or instrument sources such as electrode skin resistance, respiration, and amplifiers thermal drift. It is a low-frequency noise. In preprocessing stage, the ECG signal is filtered using the moving average filter to eliminate the baseline wander. This process is equivalent to LPF with the response of the smoothing given using "Eq. (1)".

$$
y(i)=\frac{1}{2 N+1}(x(i+N)+x(i+N-1)+\ldots+x(i-N))
$$

Where, $\mathrm{y}(\mathrm{i})$ is the smoothed value for the $\mathrm{i}^{\text {th }}$ data point, $\mathrm{N}$ is the number of neighboring data points on either side of $y(i), 2 N+1$ is the span and $x$ is input vector [26]. 
Figure 8 depicts an original ECG signal along with its noise, which has the offset of 0.5. Figure 9 depicts the baseline eliminated ECG signal, which has the offset of 0 .

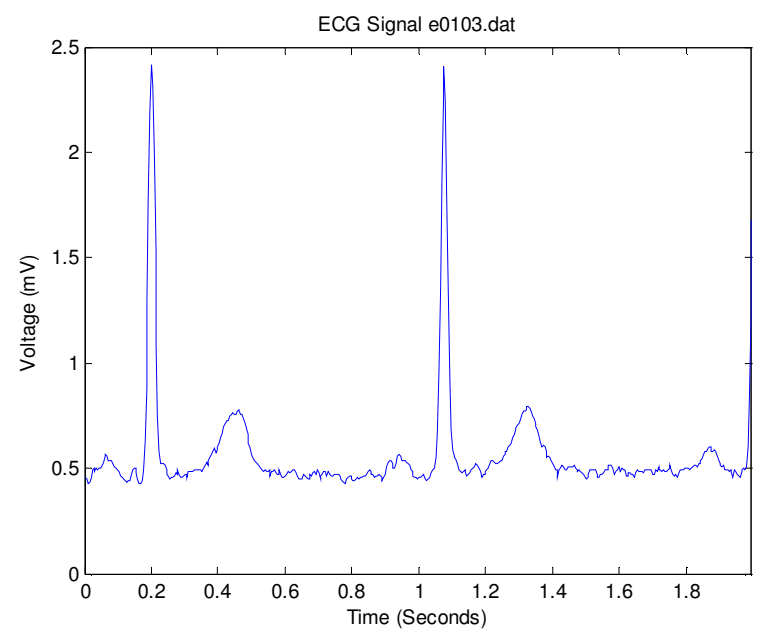

Figure 8. Original ECG signal with baseline noise which has the offset of $\mathbf{0 . 5}$.

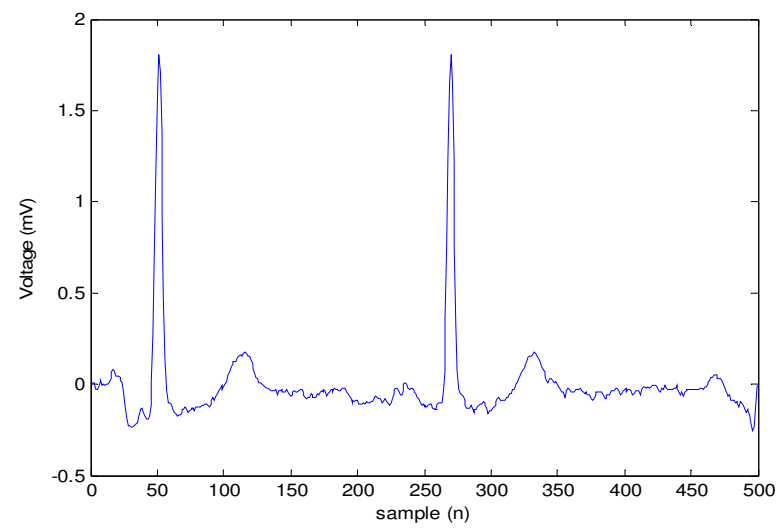

Figure 9. Baseline eliminated ECG signal which has the offset of $\mathbf{0}$

In the processing stage, the ECG features are extracted using selecting $2 \mathrm{Sec}$ of an ECG records. For feature extraction stage, we used DWT. As already mentioned there are many wavelet filters to apply on a signal. We have selected the Daubechies wavelet of order 6 (db6). This is because the Daubechies wavelet family is similar in shape to QRS complexes and their energy spectra are concentrated around low frequencies. The number of decomposition levels was set to 8 . In other words, the ECG signals have been decomposed into the details D1-D8. In order to reduce the dimensionality of the extracted feature vectors, 24 statistics over the set of the wavelet coefficients were used from first level to eighth level. The sets of features are given bellow:

- Maximum of the wavelet coefficients in each level.

- Minimum of the wavelet coefficients in each level.

- Variance of the wavelet coefficients in each level. 


\subsection{Classification Phase}

In the classification phase, we have used an MLP neural network. The best architecture of the MLP NN is usually obtained using a trial-and-error process [27], [28]. Therefore, after running many simulations, we chose an MLP NN with 24 input neurons, one hidden layer, and 2 linear output neurons. Bipolar outputs using +1 and -1 numbers were used as the output target for the three selected classes. The performance of the proposed MLP NN was tested using the MeanSquared Error (MSE) parameter. This error is computed using the differences between the actual outputs and the outputs obtained by the trained NN.

In the neural network model that has been implemented using MATLAB programming tools, there were several training algorithms which have a variety of different computation and storage requirements. However, it is hard to find an algorithm that is well suited to all applications. In our works, we tried to implement our algorithm using several high-performance algorithms such as Variable Learning Rate (or "traingdx"), Resilient Backpropagation (or "trainrp") and Reduced Memory Levenberg-Marquardt (or "trainlm") algorithms as the training algorithm. Two bipolar outputs were used as the target of network. The targets for three classified patterns are: [1,1] for normal signal, $[-1,-1]$ for PB arrhythmia, $[-1,1]$ for APB arrhythmia. Heuristic techniques are used by traingdx and trainrp algorithms. The heuristic techniques were developed using an analysis of the performance of the standard steepest descent algorithm. The trainlm algorithm uses standard numerical optimization techniques [29]. The training curve for proposed MLP neural network is depicted in Figure 10. As can be seen in the figure, the best and goal training curve are overlapped.

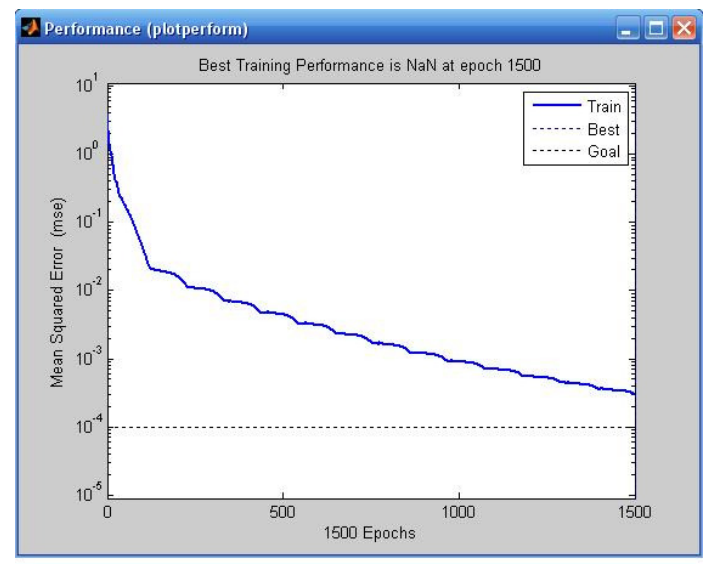

Figure 10. Training curve for proposed MLP neural network.

\section{EXPERIMENTS AND SIMULATION RESULTS}

The MIT-BIH arrhythmia database consists of 48 ECG signal records. Each record comprises several files, the signals, annotations and specifications of signal attributes. Each record of the MIT-BIH database is 30 minutes selected from 24 hours. The sampling frequency of the ECG signals in this database is $360 \mathrm{~Hz}$, and records are annotated throughout; by this we mean that each beat is described by a label called an annotation. Typically an annotation file for an MIT$\mathrm{BIH}$ record contains about 2000 beat annotations, and smaller numbers of rhythm and signal quality annotations. 
In this study, we used 10 records from this database that is listed in Table 1 and we selected 500 samples from each record.

Table 1. Selected records from database.

\begin{tabular}{|c|c|}
\hline Class & Record numbers \\
\hline Normal & $103-115-121-123$ \\
\hline PB & $104-107-217$ \\
\hline APB & $209-220-223$ \\
\hline
\end{tabular}

The proposed MLP neural networks were trained using 90 training vectors from 10 files of the MIT-BIH arrhythmia database. The trained MLP NNs were tested using 45 patterns (15 testing patterns for each class) using 10 files including normal and two arrhythmias. In order to test the performance of the trained MLP NN, We have used two criteria to compare the trained networks, recognition samples and recognition rate. Recognition rate is defined as follows:

$$
A=100\left(\frac{N c}{N t}\right)
$$

Where $\mathrm{A}$ is the recognition rate, $\mathrm{Nc}$ is the number of correctly classified patterns, and $\mathrm{Nt}$ represents the total number of patterns.

The simulation results are depicted in Table 2, 3 and 4.The test results obtained by training the proposed MLP NN using different number of neurons in the hidden layer.

Table 2 shows the results which have been obtained using training traingdx algorithm. We use 12, 13, and 14 neurons in the hidden layer. As can be seen the best performance is obtained using 13 neurons. The proper setting of the learning rate for traingdx algorithm is very important. This is because its performance is very sensitive to the proper setting of this rate. For example, if the learning rate is set too high, the algorithm can oscillate and it became unstable, while if the learning rate is too small, the algorithm takes too long to converge. It is hard to determine and obtain the optimal setting for this variable before trainings phase. This is because during the training process, the optimal learning rate is changed.

Table 3 depicts results which have been calculated using training trainrp algorithm. It uses 10, 11, and 12 neurons in the hidden layer. The best performance was obtained by using 11 neurons in the hidden layer. The gradient can have a very small magnitude when the steepest descent has been used to train the ANN with sigmoid functions. This leads small changes in the biases and the weights, even though the biases and weights are far from their optimal values. The goal of the trainrp algorithm is to remove these harmful effects of the magnitudes of the partial derivatives. The obtained results using training trainlm algorithm is depicted in Table 4. We got the best performance using 14 neurons among 13, 14, and 15 neurons in the hidden layer. This algorithm is the fastest algorithm but the main drawback of this algorithm is that it requires the storage of some matrices that can be quite large for certain problems. In addition, the best recognition rate of the trained MLP NN with three train algorithms is $96.5 \%$. 
International Journal of Computer Science, Engineering and Applications (IJCSEA) Vol.2, No.1, February 2012

In addition, Table 5 summarizes the results obtained by the other algorithms proposed in the literature. As it can be seen in Table 5, our proposed algorithm has high performance in compared to some other algorithms for ECG arrhythmias classification.

Table 2. Simulation result with traingdx algorithm.

\begin{tabular}{|c|c|c|c|c|c|c|c|c|}
\hline & & & \# or & n net & & \# of & n neu & \\
\hline D & & \# of & 12 & 13 & 14 & 12 & 13 & 14 \\
\hline & & & $\operatorname{Re}$ & $d s a n$ & & Recc & n rat & \\
\hline Nomm & Train & 30 & 30 & 30 & 30 & 100 & 100 & 100 \\
\hline & Test & 15 & 12 & 13 & 13 & 80 & 86 & 86 \\
\hline PD & Train & 30 & 30 & 30 & 30 & 100 & 100 & 100 \\
\hline & Test & 15 & 15 & 15 & 15 & 100 & 100 & 100 \\
\hline ג & Train & 30 & 30 & 30 & 30 & 100 & 100 & 100 \\
\hline & Test & 15 & 13 & 14 & 13 & 86 & 93 & 86 \\
\hline & & 135 & 130 & 132 & 131 & 94.3 & 96.5 & 95.3 \\
\hline
\end{tabular}

Table 3. Simulation result with trainrp algorithm.

\begin{tabular}{|c|c|c|c|c|c|c|c|c|}
\hline \multirow{3}{*}{\multicolumn{2}{|c|}{ Data }} & \multirow{3}{*}{$\begin{array}{c}\# \text { of } \\
\text { samples }\end{array}$} & \multicolumn{3}{|c|}{ \# of hidden neurons } & \multicolumn{3}{|c|}{ \# of hidden neurons } \\
\hline & & & 10 & 11 & 12 & 10 & 11 & 12 \\
\hline & & & \multicolumn{3}{|c|}{ Recognized samples } & \multicolumn{3}{|c|}{ Recognition rate (\%) } \\
\hline \multirow{2}{*}{ Normal } & Train & 30 & 30 & 30 & 30 & 100 & 100 & 100 \\
\hline & Test & 15 & 12 & 13 & 12 & 80 & 86 & 80 \\
\hline \multirow{2}{*}{ PB } & Train & 30 & 30 & 30 & 30 & 100 & 100 & 100 \\
\hline & Test & 15 & 15 & 15 & 15 & 100 & 100 & 100 \\
\hline \multirow{2}{*}{ APB } & Train & 30 & 30 & 30 & 30 & 100 & 100 & 100 \\
\hline & Test & 15 & 13 & 14 & 14 & 86 & 93 & 93 \\
\hline \multicolumn{2}{|c|}{ Total } & 135 & 130 & 132 & 131 & 94.3 & 96.5 & 95.5 \\
\hline
\end{tabular}

Table 4. Simulation result with trainlm algorithm.

\begin{tabular}{|c|c|c|c|c|c|c|c|c|}
\hline \multirow{3}{*}{\multicolumn{2}{|c|}{ Data }} & \multirow{3}{*}{$\begin{array}{c}\text { \# of } \\
\text { samples }\end{array}$} & \multicolumn{3}{|c|}{ \# of hidden neurons } & \multicolumn{3}{|c|}{ \# of hidden neurons } \\
\hline & & & 13 & 14 & 15 & 14 & 15 & 16 \\
\hline & & & \multicolumn{3}{|c|}{ Recognized samples } & \multicolumn{3}{|c|}{ Recognition rate (\%) } \\
\hline \multirow{2}{*}{ Normal } & Train & 30 & 30 & 30 & 30 & 100 & 100 & 100 \\
\hline & Test & 15 & 13 & 13 & 12 & 86 & 86 & 80 \\
\hline
\end{tabular}


International Journal of Computer Science, Engineering and Applications (IJCSEA) Vol.2, No.1, February 2012

\begin{tabular}{|c|c|c|c|c|c|c|c|c|}
\hline \multirow{2}{*}{ PB } & Train & 30 & 30 & $\mathbf{3 0}$ & 30 & 100 & $\mathbf{1 0 0}$ & 100 \\
\cline { 2 - 9 } & Test & 15 & 15 & $\mathbf{1 5}$ & 10 & 100 & $\mathbf{1 0 0}$ & 100 \\
\hline \multirow{2}{*}{ APB } & Train & 30 & 30 & $\mathbf{3 0}$ & 30 & 100 & $\mathbf{1 0 0}$ & 100 \\
\cline { 2 - 9 } & Test & 15 & 13 & $\mathbf{1 4}$ & 13 & 86 & $\mathbf{9 3}$ & 86 \\
\hline \multicolumn{2}{|c|}{ Total } & 135 & 131 & $\mathbf{1 3 2}$ & 130 & 95.3 & $\mathbf{9 6 . 5}$ & 94.3 \\
\hline
\end{tabular}

Table 5. Comparison between other related algorithms and our proposed algorithm.

\begin{tabular}{|c|c|c|c|c|}
\hline Authors & Method & Data base & Arrhythmia types & $\begin{array}{c}\text { Accuracy } \\
(\%)\end{array}$ \\
\hline $\begin{array}{l}\text { (E. D. Ubeyli, } \\
\text { 2008)[25] }\end{array}$ & $\begin{array}{l}\text { discrete wavelet } \\
\text { transform / mixture } \\
\text { of experts network } \\
\text { structures }\end{array}$ & $\begin{array}{c}\text { (Physiobank } \\
\text { database) }\end{array}$ & $\begin{array}{c}\text { normal beat, } \\
\text { congestive heart } \\
\text { failure beat, } \\
\text { ventricular } \\
\text { tachyarrhythmia beat, } \\
\text { atrial fibrillation beat, } \\
\text { partial epilepsy beat }\end{array}$ & 96.89 \\
\hline $\begin{array}{l}\text { (T. Inan } \\
\text { Omer, L. } \\
\text { Giovangrandi, } \\
\text { and T. A. } \\
\text { Kovacs } \\
\text { Gregory, } \\
\text { 2006)[30] }\end{array}$ & $\begin{array}{l}\text { Combining wavelet } \\
\text { and timing interval / } \\
\text { neural network }\end{array}$ & $\begin{array}{c}22 \text { files } \\
\text { (MIT-BIH } \\
\text { arrhythmias } \\
\text { data base) }\end{array}$ & $\begin{array}{c}\text { PVC - Normal - } \\
\text { Other beat }\end{array}$ & 95.16 \\
\hline $\begin{array}{l}\text { (M. Engin, } \\
\text { 2004)[31] }\end{array}$ & $\begin{array}{l}\text { wavelet transform } \\
\text { variance, third-order } \\
\text { cumulant and AR } \\
\text { model/fuzzy-cmeans } \\
\text { classifier and MLP } \\
\text { neural network }\end{array}$ & $\begin{array}{c}4 \text { files } \\
\text { (MIT-BIH } \\
\text { arrhythmias } \\
\text { data base) }\end{array}$ & $\begin{array}{l}\text { Normal, Non- } \\
\text { conducted P wave, } \\
\text { Premature ventricular } \\
\text { contraction beats, and } \\
\text { Right bundle branch } \\
\text { block beats }\end{array}$ & 98 \\
\hline $\begin{array}{l}\text { (H. G. } \\
\text { Hosseini, K. } \\
\text { J. Reynolds, } \\
\text { and D. } \\
\text { Powers, } \\
\text { 2001)[32] }\end{array}$ & $\begin{array}{c}\text { Multi stage Neural } \\
\text { network }\end{array}$ & $\begin{array}{c}10 \text { files } \\
\text { (MIT-BIH } \\
\text { arrhythmias } \\
\text { data base) }\end{array}$ & $\begin{array}{c}\text { Normal - PVC }-\mathrm{PB} \\
-\mathrm{RBBB}- \\
\text { artial premature beat } \\
\text { - fusion of paced and } \\
\text { normal beat }\end{array}$ & 88.33 \\
\hline This work & DWT and NN & $\begin{array}{c}10 \text { files } \\
\text { (MIT-BIH } \\
\text { arrhythmias } \\
\text { data base) }\end{array}$ & Normal - PB - APB & 96.5 \\
\hline
\end{tabular}




\section{Conclusions}

In this paper, a neural network based system for automatic ECG arrhythmias classification was proposed. We have used 10 recordings from the MIT-BIH arrhythmias database for training as well as testing our classifier. The proposed system consists of two phases: the feature extraction phase and the classification phase. In the first phase, moving average filter is employed to eliminate the baseline noise from the ECG signals. Then the DWT is applied on filtered signal and some features from the wavelet coefficients are extracted. In the second phase, the extracted features are used to train an MLP NN as the classifier. The simulation results demonstrated the proposed system could be employed for the classification of the ECG arrhythmias with a recognition rate of $96.5 \%$, when 13 neurons were in the hidden layer in traingdx, 11 neurons in trainrp algorithm and 14 neurons in trainlm.

\section{REFERENCES}

[1] R. Acharya, P. S. Bhat, S. S. Iyengar, A. Roo and S. Dua, (2002) "Classification of heart rate data using artificial neural network and fuzzy equivalence relation", The Journal of the Pattern Recognition Society, vol. 130, pp. 101-108.

[2] K. Minami, H. Nakajima and T. Toyoshima, (1999) "Real-Time discrimination of ventricular tachyarrhythmia with fourier-transform neural network", IEEE Trans. on Biomed. Eng, vol. 46, pp. 179-185.

[3] I. Romero and L. Serrano, (2001) "ECG frequency domain features extraction: A new characteristic for arrhythmias classification", in Proc.23rd Annual Int. Conf. on Engineering in Medicine and Biology Society, pp. 2006-2008.

[4] P. de Chazal, M. O'Dwyer and R. B. Reilly, (2000) "A comparison of the ECG classification performance of different feature sets", IEEE Trans. on Biomed. Eng, vol. 27, pp. 327-330.

[5] P. de Chazal, M. O'Dwyer and R. B. Reilly, (2004) "Automatic classification of heartbeats using ECG morphology and heartbeat interval features”, IEEE Trans. on Biomed. Eng, vol. 51, pp. 11961206.

[6] C. Alexakis, H. O. Nyongesa, R. Saatchi, N. D. Harris, C. Davis, C. Emery, R. H. Ireland and S. R. Heller, (2003) "Feature extraction and classification of electrocardiogram (ECG) signals related to hypoglycemia", Proc. Computers in Cardiology, vol. 30, pp. 537-540.

[7] P. Ivanov, M. QDY, R. Bartsch, et al, (2009) "Levels of complexity in scaleinvariant neural signals", Physical Review.

[8] S. Z. Mahmoodabadi, A. Ahmadian, M. Abolhasani, P. Babyn and J. Alirezaie, (2010) "A fast expert system for electrocardiogram arrhythmia detection”, Expert system, vol.27, pp. 180-200.

[9] S. Osowski, L. T. Hoai and T. Markiewicz, (2004) "Support vector machine-based expert system for reliable heartbeat recognition", IEEE Trans. on Biomed. Eng, vol. 51, No. 4, pp. 582-589.

[10] T. H. Linh, S. Osowski and M. Stodolski, (2003)“On-line heart beat recognition using hermite polynomials and neuro-fuzzy network”, IEEE Trans. on Instrumentation and Measurement, vol. 52, pp. 1224-1231.

[11] Y. H. Hu, W. J. Tompkins, J. L. Urrusti and V. X. Afonso,( 1994) "Applications of artificial neural networks for ECG signal detection and classification”, The Journal of Electrocardiology, vol. 26, pp. 66-73.

[12] Y. Hu, S. Palreddy and W. J. Tompkins,( 1997) "A patient-adaptable ECG beat classifier using a mixture of experts approach", IEEE Trans. on Biomed. Eng, vol. 44, pp. 891-900.

[13] S. B. Ameneiro, M. Fernández-Delgado, J. A. Vila-Sobrino, C. V. Regueiro and E. Sánchez, (1998) “Classifying multichannel ECG patterns with an adaptive neural network”, IEEE Engineering in Medicine and Biology, vol. 17, pp. 45-55.

[14] M. Fernández-Delgado and S. B. Ameneiro, (1998) "MART: A Multichannel ART-based neural network", IEEE Trans. on Neural Network, vol. 9, pp. 139-150.

[15] D. A. Coast, R. M. Stern, G. G. Cano and S. A. Briller, (1990) "An approach to cardiac arrhythmia analysis using hidden markov models”, IEEE Trans. on Biomed. Eng, vol. 37, pp. 826-836. 
[16] R. V. Andreao, B. Dorizzi and J. Boudy, (2006) "ECG signal analysis through hidden markov models", IEEE Trans. on Biomed. Eng, vol. 53, pp. 1541-1549.

[17] M. Lagerholm, C. Peterson, G. Braccini, L. Edenbrandt and L. Sörnmo, (2000) "Clustering ECG complexes using hermite functions and self-organizing maps", IEEE Trans. on Biomed. Eng, vol. 47, pp. 838-848.

[18] S. Osowski, T.H. Linh, (2001) "ECG beat recognition using fuzzy hybrid neural network", IEEE Trans. Biomed. Eng., Vol. 48, pp. 1265-1271.

[19] L. Shyu, W. Hu, (2008) "Intelligent Hybrid Methods for ECG Classification-A Review," Journal of Medical and Biological Eng., Vol. 28, pp.1-10.

[20] A. Mertins,( 1999) Signal analysis wavelets, filter Banks, time-frequency transforms and applications, Wollongong.

[21] M. Jansen and P. Oonincx, (2005) Second generation wavelets and applications, Springer.

[22] S. Mallat, (1989) "A theory for multiresolution signal decomposition: the wavelet representation", IEEE Pattern Anal. and Machine Intell Ii, pp. 674-693.

[23] G. Strang and T. Nguyen, (1996) Wavelets and filter banks. Wellesley Cambridge Press.

[24] M. Misiti, Y. Misiti and G. Oppenheim, J-M. Poggi, (2006) Wavelet toolbox for use with MATLAB.

[25] E. D. Ubeyli, (2008) "Implementing wavelet transform/mixture of experts network for analysis of electrocardiogram beats", Expert system, Vol. 25, pp. 150-162.

[26] (2006) Digital signal processing toolbox user's guide for use with MATLAB7.

[27] Fausett, (1994) Fundamentals of neural networks, Prentice Hall, New Jersy.

[28] S.Heykin, (1999) Neural networks: a comprehensive foundation, Prentice Hall, New Jersy.

[29] H. Demuth, M. Beale and M. Hagan, (2006) Neural network toolbox For Use with MATLAB.

[30] T. Inan Omer, L. Giovangrandi, and T. A. Kovacs Gregory, ( 2006) "Robust neural-network-based classification of Premature Ventricular Contractions using wavelet transform and timing interval features", IEEE Trans. on Biomed. Eng, Vol. 53, pp. 2507- 2515.

[31] M. Engin, (2004) "ECG beat classification using neuro-fuzzy network", Pattern Recognition Letters, Vol. 25, pp. 1715-1722.

[32] H. G. Hosseini, K. J. Reynolds, and D. Powers, (2001) "A multi-stage neural network classifier for ECG events”, In Proc. of 23rd Int. Conf of IEEE EMBS, Vol. 2, pp.1672-1675.

[33] http://library.med.utah.edu/kw/ecg/mml/ecg_533.html

[34] http://www.neurosolutions.com

\section{Authors:}

A. Shahbahrami is an assistant professor in Department of Computer Engineering at the University of Guilan, Rasht, Iran. He got his $\mathrm{PhD}$ degree from Delft University of Technology, The Netherlands in 2008. His research interests include advanced computer architecture, image and video processing, signal processing, reconfigurable computing, parallel processing, and SIMD programming. E-mail: shahbahrami@guilan.ac.ir, asadshahbahrami@gmail.com

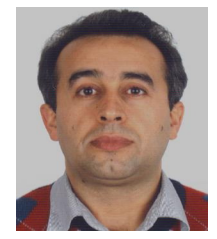

M. Kiani is an M.Sc. student in Information Technology at the University of Guilan, Iran. She received her B.Sc degree in computer science in 2009. Her research interests include signal processing, e-Learning, security in e-learning, m-learning and e-commerce. She may be reached at maedeh.kiani@gmail.com 